\title{
Based on the Enterprise Exchange Network Path Reliability Design
}

\author{
Hongxiang Zhu \\ Shangdong Woman University \\ mailjsj@163.com
}

\begin{abstract}
In order to improve the work of a single path network reliability failure case, this chapter put forward a set of a single backup path algorithm based on the path. In this algorithm, using two disjoint paths as work path to transmit data, and USES the path does not intersect with the job of a set the path as the backup path. Then, this algorithm, is proposed based on a single - a single backup path algorithm of shortest path set to ensure data under the case of the second work path of effective transmission. Theoretical derivation and numerical simulation results show that in a single work path failure cases, and the calculation of DMP compared to BP algorithm, this scheme can greatly improve network reliability, and save the network resources.
\end{abstract}

Keywords: enterprise exchange network; Reliability; Path design and cost

\section{Introduction}

With the acceleration of the society informatization process, not only users of computer network in the increase, and scale of computer network connection area and the network connection is also expanded rapidly. Information transmission, office, marketing, service, communication, entertainment and other activities can be done through the network. In both civilian and military requirement for it is becoming more and more high, in the future information war, will permeate all sorts of means and battle command, weapon system, and put them in the form of network is an organic whole repeatedly, the network will also be a combat command center and the enemy first target. In recent years, the computer network obtained the huge development, expand unceasingly, at the same time, the network reliability and network information security and confidentiality of research due to the large of network becomes more and more complex, far behind the development of the network ${ }^{[1-3]}$. Therefore, how to design the high reliability of the network and how to efficiently and accurately to the existing network complete, real reliability evaluation has become more and more important.

As the data transmitted through a set of disjoint path transmission time significantly less than the data transmitted through a single path transmission time, multipath transmission mechanism has been used in many computer networks, such as mobile AD hoc network and multi-protocol label switching network, however, multipath transmission network is easy to be affected by the failure, the failure of one or more work path, will influence the overall network normal data transmission, and could lead to the failure of the whole system. Because of the work path may be failure, need to take protective measures to ensure the effective transmission of data. A common protection measures is to use alternate paths. Sohn et al., this paper proposes a scheme of distributed label switching path to find the shortest path of the network construction cost minimum set (working path and a backup path). Article puts forward the data by $\mathrm{k}$ 
$(k \geq 2)$ the disjoint paths for transmission at the same time, among them, the disjoint paths is to point to two path does not contain the same link. In literature $[3 ; 4 ; 5]$, the data by $\mathrm{k}(k \geq 2)$ of disjoint paths for transmission at the same time, and USES the other $\mathrm{k}(k \geq 2)$ of disjoint paths (link disjoint paths) and work as the backup path. Note that consideration is $\mathrm{k}$ work path and failure. The calculation of the transmission mechanism is only all the work path fails backup path is enabled. However, does not take into account individual work path fails, enable the backup path. With the literature [6], for example $(\mathrm{k}=2)$, Lin proposed two backup paths) based on shortest path set algorithm ignores the individual work path fails, the standby path not enabled, this will lead to network only one path to transmit data, the situation in the DMP - BP algorithm to adopt two ideas do not conform to the path to transmit data. In actual network, the work path may be a pick up a failure, there is only one working path failure probability is relatively large. Due to the failure of more than one path and work rarely happens, $\mathrm{k}$ the work path and the probability of failure is relatively small. Therefore, we should work focused on a single path fails backup path selection problem.

Discover network bottlenecks, optimize the network configuration, and further found that the network of the potential danger, more effective network performance management, provide the verification and control of network quality of service, to quantify the service provider's service quality indicators, comparison and verification, has become the main purpose of network performance research. At present, the research on network performance analysis has made great progress, but still need further according to different network, the performance of different service types to make different analysis, improve the network performance analysis can comprehensively describe the network behavior. So for network performance research is still a need to further discuss. For the network performance research, network traffic collection analysis and network analysis is one of the most important performance parameter measurement method. Network traffic is to describe the network behavior, one of the most important indicators of network planning and design, business deployment, protocol and network equipment design, the implementation of traffic engineering is very important. According to the network traffic data were collected for a long time, intuitive flow model is established, the flux distribution, to predict flow and business growth, for network capacity planning and design, the rational allocation of load balancing strategy, reduce network bottlenecks. Network performance parameters measurement analysis can make the network better service to users, enabling users to timely and important business to understand the status of the network load, bandwidth usage, accurate measurement between operators, backbone network, network performance between man and business types, proper planning, evaluation network ${ }^{[6-9]}$.

In the process of the development of network reliability research, in view of the different network characteristics and purpose, the researchers have different standard of network reliability definition, this paper according to the basic reliability and mission reliability, the meaning of the definition of network reliability inductive in the literature for the following two types of network under prescribed conditions and within the stipulated time survival ability; Network under prescribed conditions and within the stipulated time to complete the task. If the networks are abstracted simple undirected network composed of nodes and links, in the first class of the definition of network reliability, the network survivability of metrics can be divided into deterministic and random indicators. Deterministic reliability index (measure) : is the sense of graph theory of reliability index, the most representative for network and network connectivity is spent "refers to the disconnect between a pair of nodes spent all the path 
needed to get rid of the minimum number of links, connectivity refers to disconnect all the pathway between a pair of nodes needed to remove the minimum number of nodes" uncertainty measure is put forward the concept of graph theory, is calculated by graph theory algorithm, it describes the network from the perspective of network connectivity topological structure on the impact of communication network reliability and the measure is important measure of network anti-destroying ability, especially for military communication network, the network of anti-destroying ability is an important indicator, but the measure has nothing to do with the reliability of the network components, so it can't fully describe the network reliability.

\section{Related Works}

Network reliability refers to the stipulated conditions and within the stipulated time network stipulated task. One of the earliest network reliability research is Lee, the study of telecommunications switching network, because network component failures make the total transmission capacity drops, caused the call congestion, Lee will block is defined as the link failure, used on the basis of connectivity reliability measure. After the network reliability research is primarily associated with the development of computer network and the development. Especially in the last century $60 \mathrm{~s}$, many organizations and schools began to network reliability research, and the ARPANET in DARPA into real concern and development, after initially established network reliability model is in the $\mathrm{N}$ nodes and $\mathrm{M}$ of the link in the network, check obeying Poisson process information through the network latency of mathematical expectation, this period is mainly based on network connectivity as to study the standard for network reliability. After $80 \mathrm{~s}$, when the Arpanet with $1980 \mathrm{~s}$ using commercial backbone packet-switched networks such as TelentTyment comparison, found that commercial packet switching network backbone significantly more dense than Arpanet, but its network connectivity probability is very low, and link density increase is caused by the larger network load initially, which shows the capacity of the network and congestion problems, such as when defining network reliability must be considered. This problem has caused an important direction in the study of network reliability, namely network performance reliability of the research, including performance reasonable determination and calculation of reliability index. But because the network performance will be affected by component failure, information flow changes, routing strategy, and the influence of such factors as the study of this kind of problem from theoretical model to analysis method has been a difficult problem. The network complexity and randomicity of performance reliability analysis brings great difficulties, so a lot of research results is based on the simulation or statistical data analysis. With the continuous development of science and technology, in the telecommunications networks, ATM networks, SDH network, WDM optical network, computer network and wireless network optimization are involved in the design of network reliability calculation problem.

Network reliability research summed up the current of network reliability indexes can be divided into four categories: anti-destroying ability, survivability, availability, and complete. (1) anti-destroying ability of network anti-destroying ability describes the network reliability of the network under the effect of man-made destruction, refers to the network topology entirely sure, under the effect of the destruction of the ideal solution, the network's ability to stay connected. For an abstract network, the network of anti-destroying ability refers to the need to destroy a few nodes or at least a few links to interrupt the connection between the part of the node, which is pointed out that the 
difficulty of damage to a network. Anti-destroying ability index is deterministic, and network topology structure, only commonly used indicators have connection and cohesive degrees. Network anti-destroying ability is only from the perspective of graph theory, the abstract into concrete network diagram, the assumption that under the premise of node and link is reliable, the evaluation of network connectivity as a reliability index, the calculation method of complexity is NP hard. (2) the network survivability network survivability refers to the nodes or links with certain failure probability of network, under the influence of stochastic damage, can maintain network connectivity probability. Survivability is based on the knowledge of probability and graph theory, describes the random damage and the influence of network topology for network reliability. One of the most typical reliability, $\mathrm{k}$ index with end to end the reliability and the reliability. Among them, the end to end index of reliability is people care about more.

(3) the availability of the network

Network availability is to describe the network under the condition of external resources available, within the prescribed period of time at any time, in can perform the required functions. The index is a kind of based on the reliability of the network business performance indicators, it points out the network under the condition of network component failure that could satisfy the requirement of communication business performance. Initially, Barberis et al., this paper offers a simple usability metrics - weighted end to end connected probability, in which the end-to-end network traffic to the corresponding end-to-end connectivity probability weighted average of the entire network. The weighted results can be used to compare different topology structure and flow distribution of the reliability of communication network, but the index is not involved in the business performance of the network. Therefore, Barberis et al., and puts forward the network throughput than the probability of a given threshold L, better to reflect the network component failure caused by the decline in network throughput. Park and others took a network availability index based on the network transmission delay. Bonaventura introduces a for circuit-switched and packet-switched networks, network availability indicators. Aggarwal proposed the use of network capacity reliability threshold method to calculate, and puts forward the concept of weighted network reliability. As you can see, the main target is the availability of network business performance, more for Internet users, also reflects the network service performance, is a hotspot of network reliability. (4) network of complete network of sex refers to the system at the beginning of the mission availability of certain circumstances, within the prescribed task profile of any random time, normal operation of system or degradation ability to fulfil the service requirements. Complete sex is mainly composed of the size of the network equipment reliability level, the network topology and network equipment service ability, network traffic distribution and other factors. Complete concept of sex research the network to the influence of fault on the routing strategy on network, network to complete the indicators framework is proposed. To sum up, the network reliability from different angles of describes the reliability of the network. In this paper, the content of the research is mainly aimed at the availability of the network. Through the research, design and implements a network reliability based on traffic acquisition and parameter measurement analysis system, according to different service requirements, make the network reliability evaluation. Network sex refers to the system at the beginning of the mission availability of certain circumstances, within the prescribed task profile of any random time, normal operation of system or degradation ability to fulfil the service requirements. Complete sex is mainly composed of the size 
of the network equipment reliability level, the network topology and network equipment service ability, network traffic distribution and other factors.

\section{System Model}

Let $G=(N, A, L, C)$ say a corporate network, among them, $N$ said a collection of nodes $A=\left\{a_{i} \mid i=1,2, \cdots, n\right\}$ that connect the nodes of the article $\mathrm{n}$ a collection of links, $L=\left\{l_{i} \mid i=1,2, \cdots, n\right\}$ said a collection of transmission delay, $C=\left\{c_{i} \mid i=1,2, \cdots, n\right\}$ let $a_{i}$ the said article i link, $l_{i}$ transmission $a_{i}$ delay, $c_{i}$ said $a_{i}$ the cost of the transmission unit flow, $M_{i}$ the maximum capacity $a_{i}, x_{i}$ said $a_{i} ; X=\left(x_{1}, x_{2}, \cdots, x_{n}\right)$ said the current capacity, the state vector (capacity) represents the network capacity. Let $\mathrm{m}$ said MP total number, said the first $\mathrm{e}, \mathrm{e}=1,2, \ldots, \mathrm{m}$ a MP, among them, $n_{e}$ said Pe included in the total number of a link, said Pe contained in the article of the link $q=1,2, \cdots, n_{e}$. Let $\mathrm{s}$ and $\mathrm{t}$, respectively, represents the source points and lodge, $d$ represents the source points to the amount of data transmission to the host point, according to the amount of data transferred on the assigned to, $d_{e}$ said the $P_{e}$ ceiling, B and T respectively, cost and time limit.

In order to solve this problem, we made some assumptions as follows:

1. Each node failure.

2. The capacity of each link is a certain probability random distribution. The capacity of the different links are independent of each other _.

3. Each path containing two disjoint paths.

4. Follow the law of conservation of traffic network. Flow conservation law means that the total capacity of a node and out of this node, with the exception of the source point and point total flow is the same. Considering the enterprise network in time and cost constraints success reliability problem of data transmission.

Through unit said the amount of data transmission when the total transport cost, the unit $F\left(d_{e}, P_{e}\right)$ said $d_{e}$ the amount of data transmission through $P$ the transmission time required. Transmission time $T\left(d_{e}, P_{e}\right)$ is delay with the data $d_{e}$ measured by the sum of path delay. $F\left(d_{e}, P_{e}\right)$ and $T\left(d_{e}, P_{e}\right)$ by type (1) (2) find out:

$$
\begin{gathered}
F\left(d_{e}, P_{e}\right)=\sum_{q=1}^{n_{e}}\left(d_{e} c_{e q}\right) \\
T\left(d_{e}, P_{e}\right)=\sum_{q=1}^{n_{e}} l_{e q}+\left\lceil\frac{d_{e}}{\min x_{e q}}\right\rceil
\end{gathered}
$$

Among them, $\left(d_{e}, c_{e q}\right)$ said data propagation through the transfer fees $a_{e q}$, min $x_{e q}$ said Pe under the state vector $\mathrm{X}$ the capacity of the network, $\lceil y\rceil$ said the smallest integer $\lceil y\rceil \geq y$. If $\mathrm{d}$ unit of data transmitted through the $\lambda(\lambda \geq 2)$ disjoint paths $\left(P_{1}, \cdots, P_{\varsigma}, \cdots, P_{\lambda}\right)$ at the same time, can be divided into $\left(d_{1}, \cdots, d_{\varsigma}, \cdots, d_{\lambda}\right)$. Let $F\left(d,\left(P_{1}, \cdots, P_{\varsigma}, \cdots, P_{\lambda}\right)\right) \mathrm{d}$ unit data propagation through $\left(P_{1}, \cdots, P_{\varsigma}, \cdots, P_{\lambda}\right)$ the total transport cost, $F\left(d,\left(P_{1}, \cdots, P_{\varsigma}, \cdots, P_{\lambda}\right)\right)$ can be made of type (3) (4) (5) find out: 


$$
\begin{gathered}
F\left(d,\left(P_{1}, \cdots, P_{\varsigma}, \cdots, P_{\lambda}\right)\right)=\sum_{\varsigma=1}^{\lambda} F\left(d_{\varsigma}, P_{\varsigma}\right) \\
d=\sum_{\varsigma=1}^{\lambda} d_{\varsigma} \\
d_{\varsigma} \leq \bar{d}_{\varsigma}
\end{gathered}
$$

Transmission, which $d_{\varsigma}$ is a negative integer $\bar{d}_{\varsigma}$ assigned to one of the biggest amount of data $P_{\varsigma}=\left\{a_{\varsigma 1}, \cdots, a_{\varsigma n_{\varsigma}}\right\} \cdot \bar{d}_{\varsigma}$ Can be gained through the type (6) :

$$
\sum_{w=1}^{u \varsigma} l_{\varsigma}+\left\lceil\frac{\bar{d}_{\varsigma}}{\min M_{\varsigma w}}\right\rceil \leq T
$$

Because in this paper, considering the cost and time limit, $T\left(d_{e}, P_{e}\right)$ and $F\left(d,\left(P_{1}, \cdots, P_{\varsigma}, \cdots, P_{\lambda}\right)\right)$ should not exceed the time limit and expense respectively, namely

$$
T\left(d_{e}, P_{e}\right) \leq T
$$

\section{The Proposed Scheme}

This paper proposed the transmission mechanism of BP algorithm is when a single work path failure, alternate paths enabled immediately. At this point, the alternate paths will work and the other normal path to form a new path. Let BP algorithm $\left(P_{b}, P_{o}\right)$ and $\left(P_{k}\right)$ respectively in the work path and backup path. $\operatorname{Pr}_{B P}\left(S \mid P_{b} P_{o}, P_{k}\right)$ can be determined by the type (8):

$$
\operatorname{Pr}_{B P}\left(S \mid P_{b} P_{o}, P_{k}\right)=\operatorname{Pr}\left(\bar{P}_{b}\right) \operatorname{Pr}\left(S \mid P_{o}, P_{k}\right)+\operatorname{Pr}\left(\bar{P}_{o}\right) \operatorname{Pr}\left(S \mid P_{b}, P_{k}\right)
$$

In order to calculate $\operatorname{Pr}_{B P}\left(S \mid P_{b} P_{o}, P_{i} P_{j}\right)$ and $\operatorname{Pr}_{B P}\left(S \mid P_{b} P_{o}, P_{k}\right)$, according to the type (7) with type (8) can be obtained, first calculate the probability of each value, and then plug in, because of the path to failure is defined as the path of at least one link failure, $\operatorname{Pr}\left(\bar{P}_{w}\right)$ expression is as follows:

$$
\operatorname{Pr}\left(\bar{P}_{w}\right)=\left(1-\prod_{r: a_{w} \in p_{w}} \operatorname{Pr}\left(x_{r} \geq 1\right)\right)
$$

Let (d, T, B, MP) - KBPS said at the time and cost constraints, the unit of data through work path transmission of successful enterprises under the network capacity of state vector boundary point, the $\operatorname{Pr}\left(S \mid P_{o}, P_{k}\right)$ and $\operatorname{Pr}\left(S \mid P_{k}\right)$ calculation are based on (d, T, B, MP) - KBPS above results.

Under the case of the second work path, we put forward the second spare BP algorithm to find the optimal path, among them, the second backup path optimization is based on the results of the first backup path. First of all, under the case of a single work path, using BP algorithm to obtain the first backup path; Then, after working in the second failure cases, using BP algorithm to obtain the second backup path. $P_{k}$ and $P_{k k}$ respectively the first and the second backup path.

$\left\langle P_{k}, P_{k k}\right\rangle$ said the first and second order of alternate paths. In the second work path failure cases, using the BP algorithm network reliability expression $\operatorname{Pr}_{B P}\left(S \mid P_{b} P_{o},\left\langle P_{k}, P_{k k}\right\rangle\right)$ such as type (10): 


$$
\operatorname{Pr}_{B P}\left(S \mid P_{b} P_{o},\left\langle P_{k}, P_{k k}\right\rangle\right)=2 \operatorname{Pr}\left(\bar{P}_{b}\right) \operatorname{Pr}\left(\bar{P}_{o}\right) \operatorname{Pr}\left(S \mid P_{k}, P_{k k}\right)+\operatorname{Pr}\left(\bar{P}_{b}\right) \operatorname{Pr}\left(\bar{P}_{o}\right) \operatorname{Pr}_{B P}\left(S \mid P_{k}, P_{k k}\right)
$$

Step 1: type (6) work out $\bar{d}_{\varsigma}$. If $\bar{d}_{\varsigma}<\mathrm{d}$, the end of the algorithm.

Step 2. [cost limit] by type (3) find out $F\left(d_{\varsigma}, P_{\varsigma}\right)$ if $F\left(d_{\varsigma}, P_{\varsigma}\right)>\mathrm{B}$, is the end of the algorithm.

Step 3. [time limit] to find out $\mathrm{d}$ under the time limit, the 4 unit data through $P_{\mathrm{k}}$ the corresponding minimum flow $f_{k}$. That is, to find the minimum integer value $f_{k}$ satisfy the following conditions.

Step 4. $X=\left(x_{1}, x_{2}, \cdots, x_{n}\right)$ can be obtained by type (10).

\section{Simulation Results}

Article 20 the MPLS network disjoint paths known $|\mathrm{r}|=$ $\{2,3,3,3,3,4,4,4,5,6,6,6,7,8,8,9,9,9,10,10\}, D=15==0.9$ and 0.975 . And before $\mathrm{r}+1$ are positioned in one of the elements has nothing to do, in this case, we have according to the proposed algorithm, a Kre $==6$. Through Sohti put forward algorithm, can find out $=4$. Figures 1 and 2 are given respectively with $K(1<K<$ article 19$)$ work after changing the number of graphs. Figure 1 and 2, respectively, and is proved to trend of change with the increase of $\mathrm{K}$ : namely increases with the increase of $\mathrm{K}$. At that time, decreases with increasing; At that time, increases with increasing.

Because, through the type (18), available $|\Pi|=4$. Then, by type (18), available

$w_{C R-D L S P}=D /|\Pi|=15 / 4=3.75$. Therefore, to the problem of two cases of CR - DLSP its optimal work path number is 4 .

Based on time series analysis methods of the cost is low, in the process of data changes smoothly, accurate calculation, but in a large area of data jitter, calculation errors; And analysis method based on historical records, the sample parameters for the history of prior information, statistical variance to get confidence interval, whether in the flat area or data jitter, relatively accurate, but with a large number of historical samples information, after a complex calculation, cost is larger; Therefore, this system, within the range of statistics to calculate the dynamic threshold is a combination of these methods. In the areas of data changes quite gentle, enabling the price smaller dynamic threshold based on time series analysis method, the dynamic threshold value are obtained by linear regression method, the data within a large area of time change, enabling more accurate analysis method based on the historical record. Discriminant data in order to change the size of the size for each parameter specifies the data change threshold, according to the different network status, set different threshold, when the data change is less than the threshold value of lambda, linear regression method is used to get the dynamic threshold scope; When the data change is greater than the corresponding threshold lambda, variance of statistical method is used to get the dynamic threshold scope; In network busy threshold should be greater than the threshold value of network free time; This method not only avoids the instability caused by linear regression problem, but also saves computational cost. 


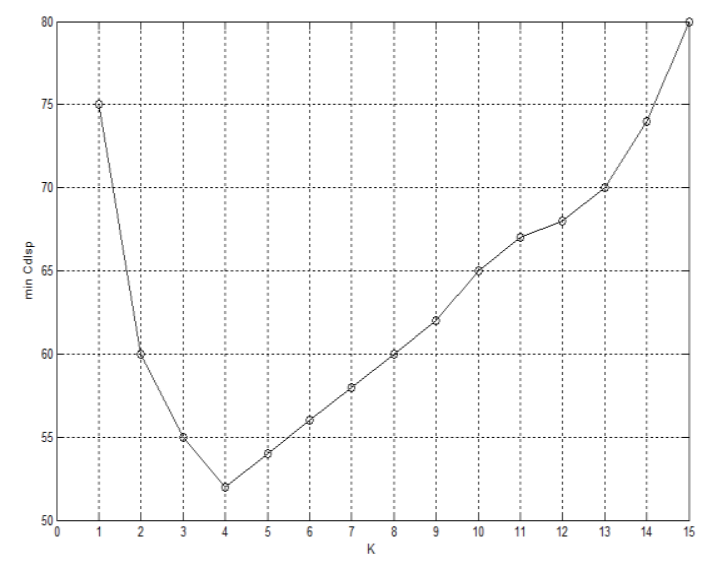

Figure 1. $\min C_{d l s p}\left(P_{K}, r_{\text {spare }}\right)$ with K Work Path Map

After working in a single failure cases, with DMP - after using BP algorithm (P4, ^ (P5) as the standby after comparison, the corresponding network reliability SMP - BP algorithm respectively to adopt a single road after P4 and P5 as alternate paths cases corresponding network reliability were improved to 0.145 , as shown in Figure 2.

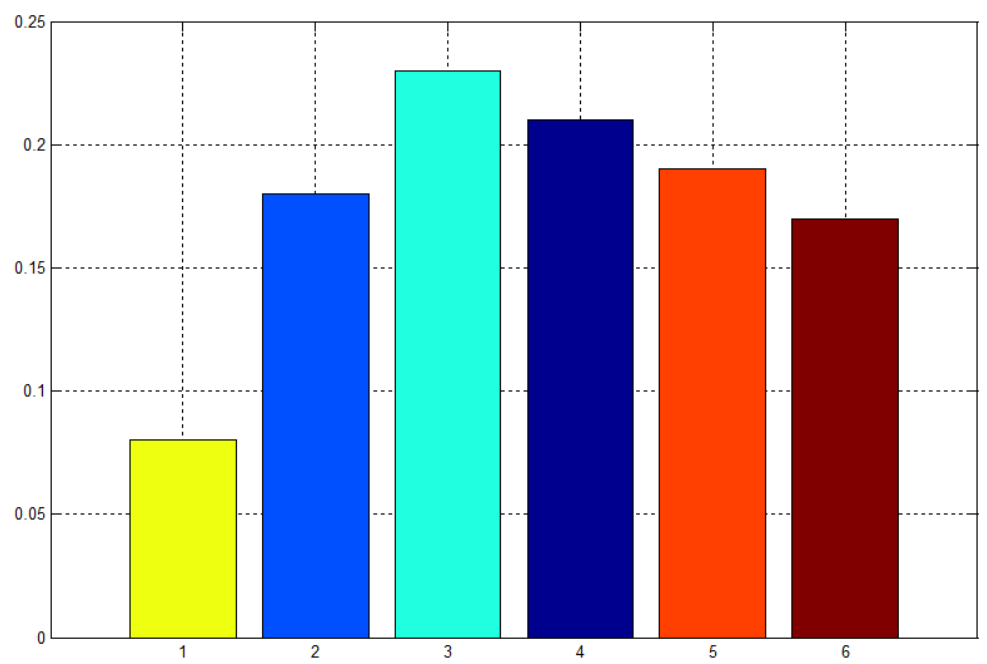

Figure 2. A Single Work POath Fails, BP Algorithm Adopts Different Alternate Paths of Network Reliability

\section{Conclusion}

This paper proposes a BP algorithm to improve the work of a single path of failure cases of network reliability. After BP algorithm adopts two disjoint paths as work path, and to adopt a path does not intersect with work path as the backup path. When a work path fails, the standby path will be enabled and immediately and the other is not the work of the failure path to form a new path to, at this time there are still two paths in a network to transmit data at the same time. Theoretical analysis and numerical simulation shows that under the case of a 
single work path, the corresponding BP algorithm network reliability saves network resources.

\title{
References
}

[1] C. C. Jane, W. H. Shen and Y. W. Laih, " Practical sequential bounds for approximating two-terminal reliability”, European Journal of Operational Research, vol. 195, no. 2, (2009), pp. 427-441.

[2] Y. K. Lin, "Spare routing reliability for a stochastic flow network through two minimal paths under budget constraint", IEEE Transactions on reliability, vol. 59, no. 1, (2010), pp. 2-10.

[3] Y. K. Lin and C. T. Yeh", Multi-objective optimization for stochastic computer networks using nsga-ii and topsis", European Journal of Operational Research, vol. 218, no. 3, (2012), pp. 735-746.

[4] C. J. Colboum, "The combinatorics of network reliability", New York: Oxford University Press, (1987).

[5] W. C. Yeh, "A simple minimal path method for estimating the weighted multi-commodity multistate unreliable networks reliability", Reliability Engineering and System Safety, vol. 93, no. 1, (2008), pp. 125136.

[6] W. C. Yeh, "An improved sum-of-disjoint-products technique for the symbolic network reliability analysis with known minimal paths", Reliability Engineering and System Safety, vol. 92, (2007), pp. 260-268S.

[7] M. J. Zuo, Z. Tian and H. Z. Huang, "An efficient method for reliability evaluation of multistage networks given all minimal path vectors", IEEE Transactions on Reliability, vol. 39, no. 8, (2007), pp. 811-817.

[8] Y. Y. K. Lin and C. T. Yeh, "Maximal network reliability with optimal transmission line assignment for stochastic electric power networks via genetic algorithms", Applied Soft Computing, vol. 1, no. 2, (2011), pp. 2714-2724.

[9] Y. K. Lin and L. C.L. Yeng, "Evaluation of network reliability for computer networks with multiple sources", Mathematical Problems in Engineering, (2012), p.18.

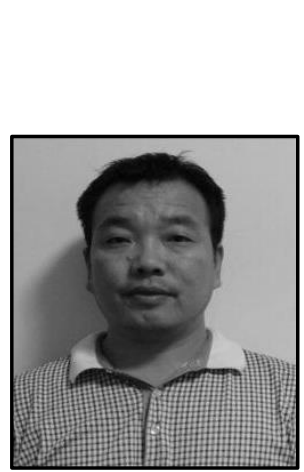

\begin{abstract}
Author
Hongxiang Zhu received the B.Eng degree in Computer Science and Technology from Shandong Institute of building materials industry the M.Eng degree in Computer Science and Technology from Shandong University, China in1997 and 2008 respectively. $\mathrm{He}$ is currently researching on the Computer Network, Electronic commerce (EC) and Management Information System (MIS).
\end{abstract}


International Journal of $u$-and e-Services, Science and Technology Vol.7, No.5 (2014) 